\title{
TB still a global killer
}

Sir - The World Health Organisation (WHO) recently claimed that the global tuberculosis situation is coming under control thanks to the development of DOTS ('directly observed treatment short-course', which is of 6 months duration $)^{1}$. The inference is that tuberculosis could be eliminated by instituting DOTS everywhere, but unfortunately that is far from the reality. There has been a drop of less than $8 \%$ in cases of tuberculosis according to $\mathrm{WHO}$, and although affluent countries such as the United States are recording real decreases, many areas of the world such as the Soviet Union are not. Tuberculosis still kills more people each year than any other single infectious disease, including malaria.

There are two main problems. The first is the delivery of medication. Supervised therapy was advocated by physicians at the Hammersmith Hospital in London as long ago as 1958 (ref. 2). Yet, in many parts of the world, adequate infrastructure for DOTS is still not in place and this requires time, commitment and money. DOTS would be simpler to introduce if the effective duration of treatment for tuberculosis was just a matter of weeks, an aim of many researching in the field.

The second but in the longer term more worrying problem is the emergence of drug resistance. Mycobacterium tuberculosis resistant to at least one commonly used drug is found in up to $20 \%$ of cases in some areas of the world, and multi-drug resistance has been well described. Some of the underlying mechanisms, such as the role of the catalase-peroxidase system in isoniazid resistance have been elucidated ${ }^{3}$, but many have not. Second-line drug therapeutic regimens are less effective, more expensive and prolonged.

Now is the time to support research to develop both true short-course therapy ( 2 weeks, not 6 months) and new immunotherapeutic approaches. If both the difficulties with DOTS and the facts of emerging drug resistance are ignored, the potential scale of the consequent disaster is large, given that $M$. tuberculosis infects approximately one third of the world's population, according to WHO figures ${ }^{4}$. It would be shortsighted to claim that the problem of tuberculosis is receding when all we may be getting from current infection control strategies is time to prepare for a more virulent pathogen. The situation remains similar to that highlighted in Nature five years ago ${ }^{5}$ which stressed the need for adequately funded, focused basic science research to combat the worldwide public health problem of tuberculosis.

\section{Jon S. Friedland}

Department of Infectious Diseases,

Royal Postgraduate Medical School,

Hammersmith Hospital,

Du Cane Road,

London W12 0NN, UK

e-mail: j.friedland@rpms.ac.uk

1. Nature 386, 534 (1997).

2. Stradling, S. \& Poole, G. Lancet ii, 1066-1067 (1958).

3. Zhang, Y. et al. Nature 358, 591-593 (1992).

4. Ravilione, M. C., Snider, D. E. \& Kochi, A. J. Am. Med. Assoc. 273 220-226 (1995).

5. Culliton, B. Nature 356, 473 (1992)

\section{Taking exception}

Sir - "It's the exception that proves the rule" is a phrase that is increasingly abused. It actually means that it's the exception that puts the rule to test, that is, studying exceptions leads to refinement and greater understanding of the rule or, alternatively, to the rule being discredited.

Unfortunately, in late twentieth-century usage, the meaning is often that it's the exception that confirms the rule, that is, any rule worth its salt ought to have a few exceptions. That is pseudoscientific, illogical, sloppy thinking, an excuse for not explaining the exception and the exact opposite of the original meaning. What, one asks, would be the consequence of applying this line of thinking to gravitation? Apples flying skyward from the tree would perhaps merely excite mild surprise as scarce exceptions to the law of universal gravitation.

I was therefore appalled to come across this phrase in a News and Views article in
Nature (K. W. Plaxco and M. Groß, 386, $657-659 ; 1997)$. The authors, discussing the finding of an exception to the dogma that the biological active state of a protein is always the folded state, say: "It is not clear how far these findings can be generalized, but certain other proteins show a similar behaviour, suggesting that FlgM is not the exception that proves the rule". But this is precisely what it is, an exception, the first of many, that demands revision of a rule.

When an expression has become so corrupt, it is time for the scientific community to disown it. The expression has already been mistranslated into Norwegian, so I fear that we must surrender it to the vocabulary of scientifically camouflaged superstition, along with 'the law of averages', and find a less ambiguous alternative.

\section{Andrew Jenkins}

A/S Telelab,

Strømdalsjordet 4,

3725 Skien, Norway

e-mail:andrew.jenkins@telelab.no
Not a good example

Sir - Harvard University has decided ex cathedra that scientific misconduct is not to be attributed to a researcher who has no experimental evidence to support statements made in published papers (Nature 386, 206; 1997). Bertrand Russell once remarked in a similar context that such a decision offers all the advantages that thievery offers over honest toil.

Erwin Klingsberg

Apt 930,

4000 Massachusetts Avenue NW,

Washington, DC 20016, USA

\section{Energy amplifier could have military uses}

Sir - Your interesting account ${ }^{1}$ of Claude Birraux's preliminary report of the November 1996 hearing at the French parliamentary office of technology assessment on Carlo Rubbia's particleaccelerator-based energy amplifier (the 'Rubbiatron') appears to overlook some essential aspects of the discussion that can be found in Birraux's report.

One of the most animated sessions of the full day-long hearing was that on nuclear proliferation. The reason is that particle accelerators are emerging as a very attractive substitute for nuclear reactors when it comes to building high-intensity neutron sources that can be used to breed large quantities of tritium, plutonium and other special materials of military interest ${ }^{2}$.

Indeed, in the most recent annual report of the French Atomic Energy Commission, the only reference to accelerator-based nuclear systems of the Rubbia type appears in the military section ${ }^{3}$. As you report, "France currently lacks a [high-current] proton accelerator". Such an accelerator would be very useful for breeding tritium ${ }^{3,4}$ and producing antimatter ${ }^{5}$ for military purposes.

So even if the 'Rubbiatron' fails to produce electricity or to dispose of nuclear waste efficiently, it could still provide a cover and a test-bed for developing highcurrent accelerators with many important military applications.

André Gsponer

Independent Scientific Research Institute,

Box 30,

1211 Geneva 12,

Switzerland

\footnotetext{
1. Glover, E. Nature 386, 426 (1997).

2. Gsponer, A. et al. Atomkernenergie-Kerntechnik 43, 169-173 (1983).

3. Rapport annuel 1995, 33, Commissariat à l'Energie Atomique July 1996.

4. Macilwain, C. Nature 377, 567 (1995).

5. Gsponer, A. \& Hurni, J.-P. Atomkernenergie-Kerntechnik 49,
} 198-203 (1987); Nature 325, 754 (1987). 